Draft VERSion March 22, 2021

Typeset using $\mathrm{LAT}_{\mathrm{E}} \mathrm{X}$ twocolumn style in AASTeX63

\title{
An optical overview of blazars with LAMOST I: Hunting changing-look blazars and new redshift estimates
}

\author{
Harold A. Peña-Herazo (D), ${ }^{1,2}$ Francesco Massaro (D) ${ }^{1,3,4,5}$ Minfeng Gu (D) ${ }^{6}$ Alessandro Paggi (D) 4,5 \\ Marco Landoni (iD, ${ }^{7,8}$ Raffaele D’Abrusco (D), ${ }^{9}$ Federica Ricci (D), ${ }^{10,11}$ Nicola Masetti (D), ${ }^{11,12}$ And \\ VAHRAM ChaVUShyan (D) ${ }^{2}$ \\ ${ }^{1}$ Dipartimento di Fisica, Università degli Studi di Torino, via Pietro Giuria 1, I-10125 Torino, Italy. \\ ${ }^{2}$ Instituto Nacional de Astrofísica, Óptica y Electrónica, Apartado Postal 51-216, 72000 Puebla, México. \\ ${ }^{3}$ Istituto Nazionale di Fisica Nucleare, Sezione di Torino, I-10125 Torino, Italy. \\ ${ }^{4}$ INAF-Osservatorio Astrofisico di Torino, via Osservatorio 20, 10025 Pino Torinese, Italy. \\ ${ }^{5}$ Consorzio Interuniversitario per la Fisica Spaziale (CIFS), via Pietro Giuria 1, I-10125, Torino, Italy. \\ ${ }^{6}$ Key Laboratory for Research in Galaxies and Cosmology, Shanghai Astronomical Observatory, Chinese Academy of Sciences, 80 Nandan \\ Road, Shanghai 200030, People's Republic of China \\ ${ }^{7}$ INAF-Osservatorio Astronomico di Cagliari, via della Scienza, 5, Selargius, CA, Italy. \\ ${ }^{8}$ INAF-Osservatorio Astronomico di Brera, Via Emilio Bianchi 46, I-23807 Merate, Italy. \\ ${ }^{9}$ Center for Astrophysics - Harvard 83 Smithsonian, 60 Garden Street, Cambridge, MA 02138, USA. \\ ${ }^{10}$ Dipartimento di Fisica e Astronomia, Università di Bologna, via P. Gobetti 93/2, I-40129, Bologna, Italy. \\ ${ }^{11}$ INAF-Osservatorio di Astrofisica e Scienza dello Spazio, via Gobetti 93/3, I-40129, Bologna, Italy. \\ ${ }^{12}$ Departamento de Ciencias Físicas, Universidad Andrés Bello, Fernández Concha 700, Las Condes, Santiago, Chile.
}

(Received March 22, 2021; Revised -)

Submitted to AJ

\begin{abstract}
The extragalactic $\gamma$-rays sky observed by Fermi-Large Area Telescope (LAT) is dominated by blazars. In the fourth release of the Fermi-LAT Point Source Catalog (4FGL), are sources showing a multifrequency behavior similar to that of blazars but lacking an optical spectroscopic confirmation of their nature known as Blazar Candidate of Uncertain type (BCUs). We aim at confirming the blazar nature of BCUs and test if new optical spectroscopic observations can reveal spectral features, allowing us to get a redshift estimate for known BL Lac objects. We also aim to search for and discover changinglook blazars (i.e., blazars that show a different classification at different epochs). We carried out an extensive search for optical spectra available in the Large Sky Area Multi-Object Fibre Spectroscopic Telescope (LAMOST) Data Release 5 (DR5) archive. We selected sources out of the 4FGL catalog, the list of targets from our follow-up spectroscopic campaign of unidentified or unassociated $\gamma$-ray sources, and the multifrequency catalog of blazars: the Roma-BZCAT. We selected a total of 392 spectra. We also compare some of the LAMOST spectra with those available in the literature. We classified 20 BCUs confirming their blazar-like nature. Then we obtained 15 new redshift estimates for known blazars. We discovered 26 transitional (i.e., changing-look) blazars that changed their classification. Finally, we were able to confirm the blazar-like nature of six BL Lac candidates. All remaining sources analyzed agree with previous classifications. BL Lac objects are certainly the most elusive type of blazars in the $\gamma$-ray extragalactic sky.
\end{abstract}

Keywords: Optical identification - Blazars - BL Lacertae objects - Flat-spectrum radio quasars 
Powered by a supermassive black hole lying in the center of elliptical galaxies, blazars are a subclass of radio-loud active galaxies mainly characterized by nonthermal emission that dominates their broad-band spectral energy distribution, although blazars can be dominated by thermal emission in optical to ultraviolet frequencies (Giommi et al. 1995; Fossati et al. 1998; Abdo et al. 2010a,b; Mao et al. 2016). They are certainly the rarest class of extragalactic radio sources (Urry \& Padovani 1995) but, on the other hand, are also the largest known population of gamma-ray objects (Acero et al. 2015; Abdollahi et al. 2020; Massaro et al. 2015c).

Blazars feature peculiar, multifrequency properties as (i) flat radio spectra both below (Massaro et al. 2013; Nori et al. 2014; Giroletti et al. 2016) and above $\sim 1 \mathrm{GHz}$ (Healey et al. 2007; Petrov et al. 2013; Schinzel et al. 2015, 2017), (ii) radio to optical polarization (Poutanen 1994; Park et al. 2018; Mandarakas et al. 2019; Liodakis \& Blinov 2019), (iii) super-luminal motion seen at radio frequencies (Jorstad et al. 2001; Kellermann et al. 2007; Lister et al. 2013), (iv) infrared colors, not simply ascribable to dust emission, (Massaro et al. 2011; D'Abrusco et al. 2012), (v) bolometric luminosities up to $10^{46-48} \mathrm{erg} / \mathrm{s}$ (Zhang et al. 2012) and (vi) both intensity and spectral variability at all frequencies from radio and optical (Gu \& Ai 2011; Chatterjee et al. 2012; León-Tavares et al. 2013; Isler et al. 2013; Hayashida et al. 2015; Patiño-Álvarez et al. 2017; Sarkar et al. 2019; Nalewajko et al. 2019; Zhang et al. 2020; Chavushyan et al. 2020) up to TeV energies (Giannios et al. 2009; Acciari et al. 2010; Archambault et al. 2015) and with daily to minutes timescales (Aharonian et al. 2007; Albert et al. 2007; Paliya et al. 2017; Liu et al. 2017; Gupta 2018), all coupled with a typical double-humped broadband spectral energy distribution (SED) (Abdo et al. 2010a; Fan et al. 2016). In 1978, at the Pittsburg conference, when the word blazar was coined, Blandford and Rees proposed the current interpretation of blazar observational properties due to relativistic particles accelerated in a plasma jet closely aligned to the line of sight (Blandford \& Rees 1978).

From an optical perspective, blazars are mainly divided into two sub-classes: BL Lac objects and flatspectrum radio quasars (FSRQs). The former sub-class shows an almost featureless spectrum with a relatively blue continuum where only weak emission or absorption lines are, rarely, superimposed and, when occurs, with an equivalent width less than $\sim 5 \AA$ (Stickel et al. 1991; Stocke et al. 1991). On the other hand, the latter sub-class present the typical quasar-like spectra with relatively intense and broad emission lines and the presence of the big blue bump peaking at ultraviolet wave- lengths (see, e.g., Wu et al. 2012; Shaw et al. 2012). According to the multifrequency catalog of blazars, the Roma-BZCAT, the former sub-class, is generally indicated as BZB, while the latter as BZQ (Massaro et al. 2009). Then in the recent release of the Roma-BZCAT (Massaro et al. 2015a), radio sources, usually reported as BL Lac objects in the literature, but showing a SED where the host galaxy emission is significantly dominant over the continuum due to the relativistic jet, are indeed labeled as BZG. The Ca II break is usually adopted to evaluate the contribution of non-thermal continuum with respect to the host emission, and thus differentiate between BZGs and BZBs (Landt et al. 2002; Massaro et al. 2012a). The Ca II break is defined as $C=\left(F_{+}-F_{-}\right) / F_{+}$, where $F_{+}$and $F_{-}$are the fluxes at rest-frame wavelengths of 3750-3950 $\AA$ and 4050-4250 A. Blazars with a $C \geq 0.25$ are classified as BZG while those with $C<0.25$ as BZB (Massaro et al. 2015a).

The spectral variability of blazars provides crucial information to study the nature of their broad line region (BLR). León-Tavares et al. (2013) reported the statistically significant flare-like event in the $\mathrm{Mg}$ II emission line in the blazar 3C 454.3 and presented direct observational evidence of the BLR close to the radio core of the jet, and this was confirmed in subsequent studies (Isler et al. 2013; Jorstad et al. 2013; Amaya-Almazán et al. 2021). Recently, similar behavior was observed for CTA 102 but on a greater scale (Chavushyan et al. 2020). These blazars are the only ones where an increase in the emission line ( $\mathrm{Mg}$ II and Fe II band) was reported. Both blazars, 3C 454.3 and CTA 102 are FSRQ type.

The last decade has undoubtedly been a golden age for blazar research, due to the all-sky survey of the Fermi satellite in the $\mathrm{MeV}-\mathrm{GeV}$ energy range, carried out thanks to its Large Area Telescope (LAT; Atwood et al. 2009). This allowed us to discover hundreds of new blazars associated with previously unidentified/unassociated $\gamma$-ray sources (UGSs; see Massaro et al. 2015c; Peña-Herazo et al. 2020, for reviews on the extragalactic sky seen by Fermi). Since the release of the First Fermi-LAT Point Source Catalog (1FGL; Abdo et al. 2010c) and until the latest, the Fourth Fermi-LAT Point Source catalog (4FGL; Abdollahi et al. 2020) it was quite clear that an almost constant fraction, about $1 / 3$, of all detected objects, were and still are UGSs (Massaro et al. 2016a), lacking an assigned low energy counterpart (Peña-Herazo et al. 2020), or being simply unclassified mainly due to the lack of an optical spectrum, recently labeled as Blazar of Uncertain type (BCUs; see also Acero et al. 2015; Ackermann et al. 2015). Hundreds of blazars were discovered thanks to new follow-up observations of UGSs and BCUs avail- 
able at radio (Kovalev 2009; Petrov et al. 2013; Massaro et al. 2013; Nori et al. 2014; Schinzel et al. 2015; Giroletti et al. 2016), optical (Paiano et al. 2017a) and X-ray (Cheung et al. 2012; Paggi et al. 2013; Acero et al. 2013; Takeuchi et al. 2013; Landi et al. 2015; Kaur et al. 2018, 2019; Marchesini et al. 2019a, 2020) frequencies as well as applying statistical methods (Ackermann et al. 2012a; Doert \& Errando 2014; Salvetti et al. 2017), hundreds of them are probably still unknown (Massaro et al. 2012c; D'Abrusco et al. 2013).

In between the releases of the Second and the Third Fermi Point Source catalogs (2FGL and 3FGL, respectively Nolan et al. 2012; Acero et al. 2015), thanks to the discovery that blazars have extremely peculiar mid-IR colors (Massaro et al. 2011; D'Abrusco et al. 2012; Massaro et al. 2012b; Massaro \& D'Abrusco 2016; D'Abrusco et al. 2019), we were able to find hundreds of potential blazar-like counterparts of UGSs (Massaro et al. 2016a; Peña-Herazo et al. 2020). Then thanks to an extensive follow up spectroscopic campaign in the optical band (Paggi et al. 2014; Massaro et al. 2015b; Landoni et al. 2015; Ricci et al. 2015; Álvarez Crespo et al. 2016a,b; Peña-Herazo et al. 2017; Marchesini et al. 2019b; PeñaHerazo et al. 2019; de Menezes et al. 2020) discovering and classifying hundreds of blazars as summarized in Peña-Herazo et al. (2020). We classified more than 400 new blazars, mainly belonging to the BL Lac subclass, the most elusive one. The results of our campaign also included a search in the archives of optical surveys (Álvarez Crespo et al. 2016c) as the Sloan Digital Sky Survey (SDSS Aguado et al. 2019) and the Six-Degree Field Galaxy Survey (6dFGS; Jones et al. 2004, 2009).

Clarifying the nature of blazar candidates and determining the redshift of the $\gamma$ blazar population is crucial to (i) determine their luminosity function (Ajello et al. 2014; Ackermann et al. 2016), (ii) study the imprint of the extragalactic background light in the blazar $\gamma$-ray spectra (e.g., Domínguez Sánchez et al. 2011; Ackermann et al. 2012b; Sandrinelli et al. 2013) (iii) select potential targets for $\mathrm{TeV}$ observatories (Massaro et al. 2013c; Arsioli et al. 2015) (iv) search for new classes of $\gamma$-rays sources (Massaro et al. 2017; Bruni et al. 2018) (v) analyze new methods for $\gamma$-rays detection (Abdollahi et al. 2020; Kerr 2019), and (vi) set constraints on the annihilation of dark matter in subhalos (see e.g., Zechlin et al. 2012; Ackermann et al. 2014; Berlin \& Hooper 2014).

Here we propose to analyze the optical spectra of several blazars samples using archival data collected by the Large Sky Area Multi-Object Fibre Spectroscopic Telescope (LAMOST, Su et al. 1998; Cui et al. 2012; Zhao et al. 2012). Our goals are to confirm the blazar nature of BCUs, to get a redshift estimate for known BL Lac objects if new optical observations can reveal the presence of spectroscopic features, and search for changing-look blazars.

We aim at exploring not only $\gamma$-ray sources classified in the 4FGL as BCUs, for which the assigned counterpart lacks an optical spectroscopic classification but also to verify the classification of all $\gamma$-ray blazars observed during our follow-up campaign and those found in the literature (see e.g., Shaw et al. 2013; Paiano et al. 2017a; Klindt et al. 2017; Desai et al. 2019) and blazars listed in the Roma-BZCAT, all lying in the LAMOST footprint. Moreover, we could detect any spectral variability between the epoch when the optical spectra were collected.

The LAMOST telescope (also known as the Guoshoujing telescope, GSJT) is a reflective Schmidt telescope with active optics with a total $5 \times 5 \operatorname{deg}^{2}$ field of view. On the focal plane, there are 4000 fiber-positioning units with a size of 3.3 arcseconds. Each unit feeds an optical fiber which transfers light to one of sixteen 250-channel spectrographs (see Su et al. 1998; Cui et al. 2012; Zhao et al. 2012, for additional details and configuration). The spectrographs have two resolution modes low resolution with $\mathrm{R} \sim 1000$ and wavelength coverage of 3700 to $9100 \AA$ and medium resolution mode with $\mathrm{R} \sim 5000$.

Archival spectra collected here are part of the widefield survey, called the LAMOST Experiment for LAMOST ExtraGAlactic Surveys (LEGAS), including as scientific objective an extra-galactic spectroscopic survey to shed light on the large scale structure of the universe (Zhao et al. 2012). LEGAS plans to acquire spectra of galaxies with magnitudes up to $r=19.5$ with a sky coverage of $11500 \mathrm{deg}^{2}$, with declination $-10<\delta<60$. We used the LAMOST data release (DR) 5 (Yao et al. 2019) that includes 152863 galaxies and 52453 quasars.

This paper is organized as follows: in Section 2 we describe our sample selection. Then, in Section 3, we present results achieved thanks to the spectral analysis of archival LAMOST spectra. Finally, Section 4 is devoted to our summary, conclusions, and future perspectives.

\section{SAMPLE SELECTION}

We extracted sources to carry out our analysis from the following three samples or catalogs.

1. The first catalog is the 4FGL (Abdollahi et al. 2020) based on the first eight years of science data collected by Fermi in the energy range between 50 $\mathrm{MeV}$ to $1 \mathrm{TeV}$. The 4FGL catalog includes 5064 sources above four sigma significance, including 75 sources that are spatially extended, 358 considered identified based on the angular extent, periodicity, 
or correlated variability observed at other wavelengths, and 1336 lacking plausible counterparts at shorter wavelengths. The 4FGL includes more than $\sim 3100$ sources, either identified or associated, with known active galaxies belonging to the blazar class and 239 pulsars.

2. We selected the 517 sources listed in our optical follow-up campaign to unveil the nature of UGSs and BCUs (Cowperthwaite et al. 2013; Paggi et al. 2014; Massaro et al. 2015b; Landoni et al. 2015; Massaro et al. 2016a; Ricci et al. 2015; Álvarez Crespo et al. 2016a,b; Peña-Herazo et al. 2017; Marchesini et al. 2019b; Peña-Herazo et al. 2019; de Menezes et al. 2020; Peña-Herazo et al. 2020). This campaign, carried out during the last decade, significantly augmented the number of known sources in the 4FGL, discovering and classifying 394 targets (see also, Massaro et al. 2013a, 2015d, 2016b; de Menezes et al. 2019) with an additional 123 sources with spectroscopic information collected from a literature search (e.g., Shaw et al. 2013; Klindt et al. 2017; Paiano et al. 2017a,b, 2019; Desai et al. 2019).

3. Finally, we also verify redshifts and classification of those blazars listed in the Roma-BZCAT v5.0 (Massaro et al. 2015a), the latest release, including a total of 3561 sources, divided as 1151 BZBs, 369 with a firm redshift estimate, 1909 BZQs, and 274 BZGs.

However, not all these sources were observed spectroscopically in the optical band, and thus not all of them have an available spectrum in the LAMOST DR 5 used in our analysis. Thus restricting to the LAMOST footprint, we extracted the following samples out of each catalog previously indicated.

1. In the 4FGL, we selected a total of 31 sources classified as one AGN, 19 BCUs, six BL Lacs, and five flat-spectrum radio quasars, the latter two classes labeled as BLL and FSRQ therein.

2. From the 517 blazars observed during the optical spectroscopic campaign of UGSs and BCUs, only eight sources have good quality spectra available in the LAMOST footprint.

3. Then, in the Roma-BZCAT, we found a total of 353 blazars investigated. These were classified as 130 BZBs, 184 BZQs, and 39 BZGs.

In those cases of sources overlapping within our three initial samples, we only indicated and list them as part of the Roma-BZCAT.
All numbers reported above did not include LAMOST spectra available with a low signal to noise ratio (i.e., below ten measured between $4000 \AA$ and $9000 \AA$ ) since they do not allow us to obtain a precise classification. It is worth noting that these numbers do not include repeated multiple matches in the considered samples, and a maximum angular separation of 2 arcsec was used to search for a LAMOST counterpart. We determined the choice of 2 arcsec as angular separation to associate each source to its LAMOST counterpart adopting the same statistical procedure described in (Massaro et al. 2014) for the blazar counterpart in the SDSS and here corresponding to a chance probability of spurious associations lower than $\sim 1 \%$.

We list all the sources from the three samples in Table 1 , along with all our results. Here we report (1) the sample each source belong to; (2) source name, (3) class and (4) redshift in the original catalog; (5) the LAMOST designated name; (6) the class and (7) the redshift identified thanks to our analysis; and, (8) a flag indicating if the source also has an available spectrum in the SDSS that we used for comparison.

\section{ANALYSIS, CLASSIFICATION AND REDSHIFT ESTIMATES}

For all sources in our three samples, we retrieved the optical spectrum available in the LAMOST DR 5 and performed the spectral analysis to measure emission or absorption lines eventually detectable. We searched for Balmer emission lines or other characteristic spectral features, as C IV, C III], Mg II, [O II], H $\beta$, [O III], $\mathrm{H} \alpha$ and $[\mathrm{N} \mathrm{II}]$ complex, or the $[\mathrm{S} \mathrm{II}]$ doublet in emission, $\mathrm{Ca}$ II H\&K, the $\mathrm{G}$ band, or $\mathrm{Mg}$ II doublet in absorption.

Data analysis was then carried out using IRAF standard packages (Tody 1986). We measured the equivalent width at the observer frame, defining a local continuum. We measured redshift for those sources we identified at least three absorption or emission lines. The line positions were estimated fitting a Gaussian profile near the peak of the line to avoid possible shifted spectral components. We estimated lower limits on the redshifts for those BZBs having detection of intervening absorption systems, as previously seen in BL Lac spectra (Stocke \& Rector 1997; Paiano et al. 2017b; Landoni et al. 2018, 2020; Paiano et al. 2020).

Since we are searching for blazars, we adopted the following classification, based on the criteria of the RomaBZCAT. We classified and labeled BL Lac objects as BZBs (featureless spectra or with emission lines of EW $<5 \AA$ ), flat-spectrum radio quasars as BZQs (quasarlike spectra), and indicated as BZGs those blazars having a non-negligible host galaxy emission in both their 
optical spectra and in their broadband SED (Massaro et al. 2012a). BZBs have almost featureless optical spectra with only weak absorption or emission lines of equivalent width less than $5 \AA$ (Stickel et al. 1991; Stocke et al. 1991), BZQs show typical quasar-like optical spectra while BZGs are more similar to classical elliptical galaxies in the optical band (Shaw et al. 2012; Massaro et al. 2012a, 2015a), same classification scheme was also used in our previous analyses (Peña-Herazo et al. 2020; de Menezes et al. 2020; Massaro et al. 2016a). In Figure 1 we show all spectra of sources belonging to each blazar class used in our analysis: a BL Lac object, a quasar-like spectrum typical of BZQs, the one of a classical elliptical galaxy as those of BZGs. In the next subsections, we describe the LAMOST spectral analysis results separately for our three catalogs (also summarized in Table 2).

\subsection{Results on the $4 F G L$ sample}

We analyzed 31 sources out of those listed in the 4FGL classified as one AGN, 20 BCUs, six BL Lacs, and four FSRQs. The AGN appear to be optically classified as a BZQ, while we classified the BCUs as four BZQs and 16 BZBs. For one BZB, we obtained a firm $z$ estimate. Then we confirm all FSRQs as BZQs and their literature redshift measurements, assuming an uncertainty of $\delta z=0.01$ for the literature estimates. We classified four out of six BL Lacs as 3 BZBs confirmed at unknown redshift, and three $\mathrm{BZB}$ confirmed at the same $z$ listed in the 4 FGL.

Then two known BL Lacs, namely: 4FGL J1410.3+1438 and 4FGL J1503.5+4759, showed a classical quasar-like spectrum leading us to label them as BZQ. Also, for one BZQ, 4FGL J1145.5+4423, we found a BZG spectrum in our analysis. These could be two transitional sources (i.e., changing look blazars), but we cannot confirm their variable nature since we could not retrieve from the literature the optical spectrum used to classify them in the $4 \mathrm{FGL}$.

\subsection{Results on targets pointed during the optical spectroscopic campaign of UGSs and BCUs}

In the sample of sources observed during our optical spectroscopic campaign, we analyzed eight optical sources distinguished as one BZQ, two BZGs, and five BZBs. The summary of our results is reported below.

We classified the BZQ (Massaro et al. 2016a) J010216.63+094411.1 as BZB, and confirmed its redshift. For all BZBs we confirm their class and redshift, four with no redshift, and one at the same $z$ previously known. The BZG J163738.24+300506.4 was originally identified as lying at $z=0.079$, however, we report that there is another source within 3 ". $^{\prime} 3$ observed in LAMOST and SDSS and we classified it as a BZQ at $z=0.819$.

The other BZG, namely J134243.61+050432.1, is indeed a radio galaxy, a.k.a. $4 \mathrm{C}+05.57$, lying at $z=0.136$ and with extended radio emission at $1.4 \mathrm{GHz}$.

\subsection{Blazars in the Roma-BZCAT}

The majority of the spectra we investigated are those related to the Roma-BZCAT for a total of 353 blazars. These were all previously classified as 184 BZQs, 39 BZGs, and 130 BZBs. The results of this part of our investigation are described in the following.

A large fraction of BZQs were confirmed in both classification and redshift estimates (i.e., assuming an uncertainty of 0.01 due to the heterogeneous uncertainties found in the literature), 174 sources out of 184 analyzed. For an additional three of them, namely: 5BZQ J1728+3838, 5BZQ J0010+1724, and 5BZQ $\mathrm{J} 1047+2635$, we provided a new redshift estimate, different from the previous one. Unfortunately, we could not find a literature spectrum to verify the $z$ estimate previously reported in the Roma-BZCAT to carry out a comparison.

We confirmed the BZG nature of 23 out of 39 BZGs analyzed, all at the same redshifts previously known. Then we found that 5BZG J0048+3157 (a.k.a. NGC 0262) shows a classical Seyfert 2-like spectrum.

Five more BZGs, namely: 5BZG J0751+1730, 5BZG J0756+3834，5BZG J1504-0248，5BZG J1512+0203, and 5BZG J2346+4024, showed a quasar-like spectrum, and we classified them as BZQs, all at the same redshifts previously known with the only exception of $5 \mathrm{BZG}$ J2346+4024 lying at $z=0.459$ instead of $z=0.0838$ as reported in the Roma-BZCAT. These five transitional (i.e., changing look) sources were found in addition to 12 BZGs for which our analysis classifies them as BZBs, one with a different redshift estimate, 5BZG J0916+5238 having a LAMOST featureless spectrum that did not allow us to confirm its previous $z=0.190$ estimate.

Finally, for $130 \mathrm{BZBs}$, our results are summarized as follows.

- Two BZBs of them appear to be transitional sources being classified as BZQs 5BZB J1402+1559 and 5BZB J1001+2911 at the same redshift.

- Seventy-nine BZBs with no redshift estimate were all confirmed, but for six cases, we also obtained a $z$ estimates or lower limits, namely for 5BZB $0124+3249$ at $z=0.780,5 B Z B$ 0127-0151 at $z=0.337$, and 5BZB J0910+3902 at $z=0.199$. In the case of 5BZB J1117+5355, 5BZB J1552+0850 


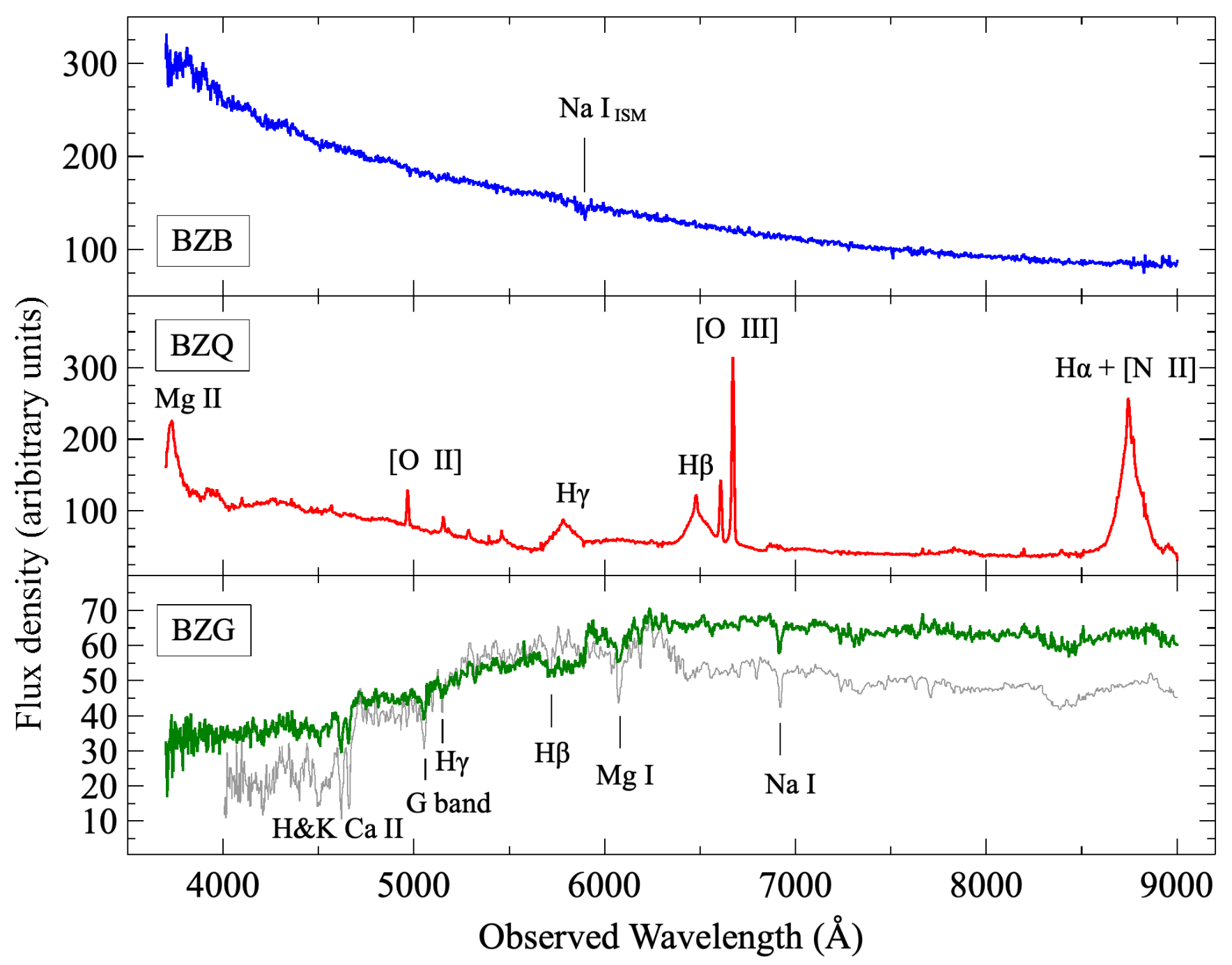

Figure 1. (Upper panel) LAMOST spectrum of J065046.49+250259.6, a classical BZB, showing the almost featureless blue spectrum, the only absorption feature is the galactic interstellar absorption of Na I. (Central panel) LAMOST spectrum of J011218.05+381856.8 a source classified as BZQ, showing a quasar-like spectrum along with labels of some emission lines. (Lower panel) In green, the spectrum of a BZG, i.e., J090536.44+470546.3 analyzed during our investigation. In grey, we compare the BZG with the spectrum of an elliptical galaxy, J084640.34+281829.6, rescaled to match the flux of the BZG at $4730 \AA$.

and 5BZB J1643+3221, the presence of intervening systems with optical features superimposed on their spectral continuum allowed us to get the following lower limits: 0.721, 1.0160 and 1.029, respectively.

- Six BZBs are labeled as BL Lac candidates in the Roma-BZCAT, but we were able to obtain a firm BZB classification and, in particular, for two of them: 5BZB J0124+3249 and 5BZB J0127-0151 also a $z$ estimate at 0.780 and 0.337 , respectively.

- We also analyzed optical spectra for 51 BZBs of the Roma-BZCAT with a known redshift esti- mate, 12 flagged as uncertain. For all these 51 BZBs, we confirmed their BL Lac nature, and two of those with uncertain redshift we could verify their $z$ values: 5BZB J1211+2242 at $z=0.453$, $5 \mathrm{BZB} \mathrm{J} 1410+2820$ at $z=0.521$, and for 5BZB $\mathrm{J} 0911+2949$, we found that it lies at $z=0.438$ instead of $z=0.446$.

\subsection{Changing-look blazars}

Given that the optical classification (BZBs, BZQs, or BZGs) is defined by an arbitrary limits on the equivalent width and continuum to spectral features contrast, their spectral variations (Gaur et al. 2012; León-Tavares 
Table 1. Classification results

\begin{tabular}{|c|c|c|c|c|c|c|c|}
\hline $\begin{array}{c}\text { Sample } \\
(1)\end{array}$ & $\begin{array}{c}\text { Name } \\
(2)\end{array}$ & $\begin{array}{c}\text { Cat. Class } \\
(3)\end{array}$ & $\begin{array}{l}\mathrm{z}_{1} \\
(4)\end{array}$ & $\begin{array}{c}\text { Designation } \\
(5)\end{array}$ & $\begin{array}{c}\text { Our Class } \\
(6)\end{array}$ & $\begin{array}{l}\mathrm{z}_{2} \\
(7)\end{array}$ & $\begin{array}{c}\text { SDSS flag } \\
(8)\end{array}$ \\
\hline BZCAT & 5BZQ J0005+0524 & $\mathrm{bzq}$ & 1.900 & J000520.21+052410.7 & $\mathrm{bzq}$ & 1.900 & $\ldots$ \\
\hline BZCAT & 5BZQ J0005+3820 & bzq & 0.229 & J000557.17+382015.1 & bzq & 0.230 & \\
\hline BZCAT & 5BZG J0006+1051 & bzg & 0.168 & J000620.30+105151.1 & bzb & 0.168 & \\
\hline BZCAT & 5BZQ J0010+1724 & bzq & 1.601 & J001033.99+172418.7 & bzq & 0.769 & \\
\hline BZCAT & 5BZB J0015+3536 & bzb & $\ldots$ & J001527.94+353639.0 & bzb & $\ldots$ & \\
\hline BZCAT & 5BZQ J0019+2602 & bzq & 0.284 & J001939.75+260252.8 & bzq & 0.284 & \\
\hline $4 \mathrm{FGL}$ & 4FGL J0021.0+0322 & bcu & $\ldots$ & J002050.26+032358.2 & bzb & $\ldots$ & $\cdots$ \\
\hline BZCAT & 5BZG J0022+0006 & bzg & 0.306 & J002200.95+000657.9 & bzb & 0.306 & $\ldots$ \\
\hline $4 \mathrm{FGL}$ & 4FGL J0028.9+3553 & bcu & $\ldots$ & J002851.97+355036.0 & bzb & $\ldots$ & $\ldots$ \\
\hline BZCAT & 5BZQ J0029+0554 & bzq & 1.314 & J002945.88+055440.6 & bzq & 1.314 & $\ldots$ \\
\hline
\end{tabular}

Note: Columns (1) Original sample; (2) Source name ; (3) class and (4) redshift in the sample catalog; (5) the LAMOST designated name; (6) class and (7) the red-shift identified thanks to our analysis; and (8) flag indicating if the source has also an available spectrum in the SDSS. This table is available in its entirety in machine-readable form.

Table 2. Summary of results

\begin{tabular}{lllllll}
\hline \hline Orig. Class & Number & BZB(z) & BZB & BZQ & BZG & Other \\
& & & & & \\
$(1)$ & $(2)$ & $(3)$ & $(4)$ & $(5)$ & $(6)$ & $(7)$ \\
\hline \multicolumn{7}{c}{4 FGL } \\
\hline AGN & 1 & $\ldots$ & $\ldots$ & 1 & $\ldots$ & $\ldots$ \\
BCU & 20 & 1 & 15 & 4 & $\ldots$ & $\ldots$ \\
BLL & 6 & 1 & 3 & 1 & 1 & $\ldots$ \\
FSRQ & 4 & $\ldots$ & $\ldots$ & 4 & $\ldots$ & $\ldots$ \\
\hline \multicolumn{7}{c}{ Optical Campaign } \\
BZQ & 1 & 1 & $\ldots$ & $\ldots$ & $\ldots$ & $\ldots$ \\
BZB & 5 & 1 & 4 & $\ldots$ & $\ldots$ & $\ldots$ \\
BZG & 2 & $\ldots$ & $\ldots$ & 1 & $\ldots$ & 1 \\
\hline \multicolumn{7}{c}{ Roma-BZCAT } \\
BZQ & 184 & $\ldots$ & 6 & 178 & $\ldots$ & $\ldots$ \\
BZB & 130 & 26 & 102 & 2 & $\ldots$ & $\ldots$ \\
BZG & 39 & 9 & 1 & 5 & 23 & 1 \\
\hline
\end{tabular}

Note: columns (1) class in the original sample (i.e. 4FGL, Roma-BZCAT or Optical Campaign); (2) total number of sources within the original class; (3) BZB with z measurements from the present analysis; (4) BZB without z measurements from the present analysis; (5) number of BZQs; (6) number of BZGs; (7) other type of AGN;

et al. 2013; Isler et al. 2013) can lead to changes in their optical classification, as reported in the literature (Vermeulen et al. 1995; Pian et al. 1999; Capetti et al. 2010; Ghisellini et al. 2011; Ruan et al. 2014; Álvarez Crespo et al. 2016a; Acosta-Pulido et al. 2017). We refer as changing-look blazars those objects with different optical spectral classifications reported in two different epochs in contrast with the definition of BL Lac adopted in other works (e.g., Shaw et al. 2012). Listing changing-look blazars is important for follow-up obser- vational programs aiming to study their spectral variation.

We compared the literature spectral classification with our classification based on LAMOST spectra. For 26 objects, we found a difference in classification. We summarize these results in Table 3. We performed a search in the literature for these potential changing-look blazars looking for their spectra and details on their classification. We found the spectra only for five of them, and we compare their literature spectra with the LAMOST one, 
see Figure 2. The five potential changing-look blazars for which we found spectra are:

- J140244.51+155956.6, originally classified BZB (Baldwin et al. 1977), in Figure 2, we show the literature spectrum Baldwin et al. (1977), in comparison with that collected from LAMOST (2013 February11) and SDSS (2007 June 21). Those spectra available in both SDSS and LAMOST shows intense $\left[\mathrm{O}_{I I}\right], \mathrm{H} \beta$ and the $\left[\mathrm{O}_{I I I}\right]$ doublet emission lines letting us classify it as a BZQ.

- 5BZG J0916+5238 is a BZG in the Roma-BzCAT, based on the spectrum of Nass et al. (1996). We reclassified it as a BZB according to our LAMOST investigation.

- 5BZQ J1321+2216 was originally classified as a BZQ by Shaw et al. (2012). The LAMOST spectrum, although with a lower signal-to-noise ratio, indicates a BZB source type. However, this difference in classification can be due to observational effects like signal-to-noise ratio.

- The spectrum of 5BZQ J1106+2812, classified as BZQ by Shaw et al. (2012), is indeed BZB according to the LAMOST spectrum. However, we can not compare it with the literature spectrum due to the gap in the LAMOST between $5200-5800$ Å. Considering this observational effect, we report it as a potential changing-look blazar.

- 5BZG J1056+0252 was reported by Fischer et al. (1998) and classified as a BZG in the RomaBzCAT. We classified it as a BZB according to the LAMOST spectrum.

The optical classification of blazars can be affected by several observational effects, including: the spectral signal-to-noise ratio, as weak spectral features can be swamp by the noise, spectral coverage, and spectral resolution, to name a few. However, despite these sources of uncertainties, on the basis of our analysis we are confident about claiming the changing-look nature of J140244.51+155956.6, J132111.20+221612.1, and J105606.61+025213.4 being less confident for the remaining ones for the reasons previously described.

Possible scenarios for accounting for the BZB-BZQ transition objects include that these blazars are:

- broad-lined objects with highly beamed jets and with radiatively efficient accretion (Ruan et al. 2014; Giommi et al. 2012),

- quasars with weak radiative cooling and with their broad lines overwhelmed by the non-thermal continuum (Ghisellini et al. 2012),
- broad-lined objects with variations of their jet bulk Lorentz factor (Bianchin et al. 2009).

We also considered the transitional objects BZB-BZG as changing-look blazars. BZGs and BZBs are differentiated by the Ca II break strength, with an arbitrary limit of $<0.25$ for BZBs. Thus changes in these classes are sensitive to the continuum arising from the jet and the orientation angle (Massaro et al. 2012a; Landt et al. 2002).

The optical classification of blazars can also be affected by selection effects. For example, as suggested by Giommi et al. (2012), intrinsically weak-lined blazars are more common in X-ray selected samples (since they are weaker in radio frequencies), while blazars with broad lines are more common in radio selected samples. The first ones are always classified as BZBs (therefore more common in X-ray limited selections), while the second ones will be classified as BZBs only if the continuum is high enough to sink the lines (therefore blazars selected in radio are more likely to change classification, since this essentially depends only on the non-thermal continuum level). Another selection effect is the lack of redshift measurements of blazars with high-synchrotron peak. Then broad-line objects are often included in the BL Lac class if their non-thermal continuum swamps the broad components (Giommi et al. 2012, 2013). An example of this variability is shown for the source 5BZB J1402+1559 that changed from BZB to BZQ showing a decrease of the continuum, that lets the line equivalent width increase when comparing the two epochs spectra. To avoid selection effects, in the literature, there are proposed classifications schemes based on a more physical basis, classifying blazars as low-ionization and high-ionization (Giommi et al. 2012, 2013; Giommi \& Padovani 2015) or equivalently by a limit on the BLR luminosity $L_{B L R} \sim 5 \times 10^{-4}$ in Eddington units, with $L_{B L R}$ the luminosity of the broad line region (Ghisellini et al. 2011).

\subsection{New Redshift measurements}

We measured the redshift for 15 blazars, ten without previous measurements reported in the literature and five for which the literature redshift do not match with our measurements. We summarize these measurements in Table 4. For three of the new measurements we could estimate lower limits, namely 5BZB J1117+5355, 5BZB $\mathrm{J} 1552+0850$, and 5BZB J1643+3221 at $\mathrm{z}>0.721$, z $>$ 1.016 , and $z>1.029$. We did not discover high redshift BZBs. All redshift estimates obtained are consistent with the known BZB's redshift distribution.

4. SUMMARY AND CONCLUSIONS 


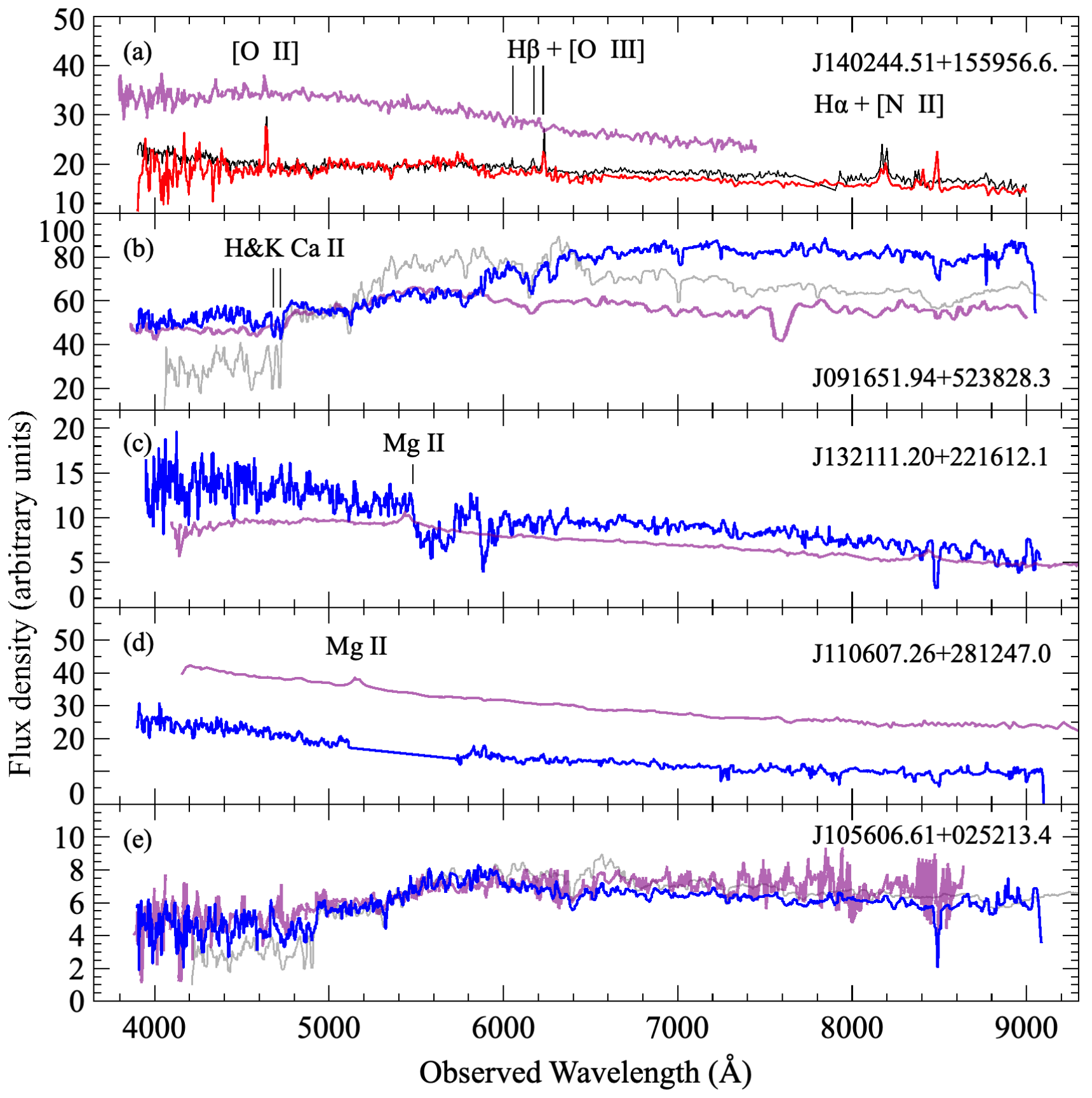

Figure 2. Comparison of the literature spectra (light violet) with the LAMOST spectra (blue if BZB red if BZQ) for those blazars with different classification with available spectral image. (a) the optical spectrum of the changing-look blazar 5BZB $\mathrm{J} 1402+1559$, in red the LAMOST spectrum, and in black the SDSS spectrum. We classified this source as BZQ and compared with the BZB spectrum reported by Baldwin et al. (1977). (b) The spectrum of 5BZG J0916+5238 classified as BZG in the Roma-BzCAT, based on the spectrum of Nass et al. (1996) compared with the BZB LAMOST spectrum. In gray, we show the spectrum of the elliptical galaxy J084640.34+281829.6 to compare the Ca II break with the blazar one. (c) The spectrum of 5BZQ J1321+2216, a BZQ as classified by Shaw et al. (2012) in comparison to the LAMOST spectrum classified here as BZB. (d) The spectrum of 5BZQ J1106+2812, classified as BZQ by Shaw et al. (2012) compared to the LAMOST spectrum showing a BZB. (e) The spectrum of the 5BZG J1056+0252 reported by Fischer et al. (1998) in comparison to the BZB LAMOST spectrum. In gray, we show the spectrum of the elliptical galaxy J084640.34+281829.6 to compare the Ca II break with the blazar one. 
Table 3. Changing-look blazars

\begin{tabular}{|c|c|c|c|c|c|}
\hline $\begin{array}{c}\text { Name } \\
(1)\end{array}$ & $\begin{array}{l}\mathrm{z}_{1} \\
(2)\end{array}$ & $\begin{array}{c}\text { Cat. Class } \\
(3)\end{array}$ & $\begin{array}{c}\text { Class Reference } \\
(4)\end{array}$ & $\begin{array}{l}\mathrm{z}_{2} \\
(5)\end{array}$ & Our Class \\
\hline \multicolumn{6}{|c|}{$4 \mathrm{FGL}$} \\
\hline 4FGL J1410.3+1438 & 0.1443 & bll & Abdollahi et al. (2020) & 0.144 & bzq \\
\hline 4FGL J1503.5+4759 & 0.3445 & bll & Abdollahi et al. (2020) & 0.344 & bzq \\
\hline \multicolumn{6}{|c|}{ Optical Campaign } \\
\hline $\mathrm{J} 134240.02+094752.4$ & 0.2828 & bzq & Álvarez Crespo et al. (2016b) & 0.283 & bzb \\
\hline \multicolumn{6}{|c|}{ Roma BzCAT } \\
\hline 5BZG J0006+1051 & 0.168 & bzg & Massaro et al. (2015a) & 0.168 & bzb \\
\hline 5BZG J0022+0006 & 0.306 & bzg & Massaro et al. (2015a) & 0.306 & bzb \\
\hline 5BZG J0303+0554 & 0.196 & bzg & Massaro et al. (2015a) & $\ldots$ & bzb \\
\hline 5BZG J0751+1730 & 0.187 & bzg & Massaro et al. (2015a) & 0.186 & bzq \\
\hline 5 BZG J0756+3834 & 0.216 & bzg & Massaro et al. (2015a) & 0.216 & bzq \\
\hline 5 BZG J0916+5238 & 0.19 & bzg & Nass et al. (1996) & 0.190 & bzb \\
\hline 5BZB J1001+2911 & 0.558 & bzb & Massaro et al. (2015a) & 0.556 & bzq \\
\hline $5 B Z Q \mathrm{~J} 1043+2408$ & 0.56 & bzq & Massaro et al. (2015a) & . & bzb \\
\hline 5BZQ J1054+3855 & 1.363 & bzq & Massaro et al. (2015a) & . & bzb \\
\hline 5BZG J1056+0252 & 0.236 & bzg & Fischer et al. (1998) & 0.239 & bzb \\
\hline 5BZG J1103+0022 & 0.275 & bzg & Massaro et al. (2015a) & 0.275 & bzb \\
\hline 5BZQ J1106+2812 & 0.844 & bzq & Shaw et al. (2012) & & bzb \\
\hline 5BZQ J1243+4043 & 1.518 & bzq & Massaro et al. (2015a) & & bzb \\
\hline 5BZQ J1321+2216 & 0.943 & bzq & Massaro et al. (2015a) & $\ldots$ & bzb \\
\hline 5BZG J1326+1229 & 0.204 & bzg & Massaro et al. (2015a) & 0.206 & bzb \\
\hline 5BZQ J1343+2844 & 0.905 & bzq & Evans \& Koratkar (2004) & $\ldots$ & bzb \\
\hline 5BZB J1402+1559 & 0.244 & bzb & Baldwin et al. (1977) & 0.245 & bzq \\
\hline 5BZG J1449+2746 & 0.227 & bzg & Massaro et al. (2015a) & 0.227 & bzb \\
\hline 5BZG J1504-0248 & 0.217 & bzg & Sabbey et al. (2001) & 0.217 & bzq \\
\hline 5BZG J1512+0203 & 0.22 & bzg & Hewitt \& Burbidge (1991) & 0.220 & bzq \\
\hline 5BZG J1730+3714 & 0.204 & bzg & Massaro et al. (2015a) & 0.205 & bzb \\
\hline 5BZG J1733+4519 & 0.317 & bzg & Massaro et al. (2015a) & 0.317 & bzb \\
\hline 5BZG J2346+4024 & 0.0838 & bzg & Sowards-Emmerd et al. (2005) & 0.459 & bzq \\
\hline
\end{tabular}

Note: Columns (1) Source name ; (2) redshift in the sample catalog; (3) redshift reference; and,(4)redshift identified thanks to our analysis. 
Table 4. New or updated redshift measurements

\begin{tabular}{|c|c|c|c|}
\hline $\begin{array}{c}\text { Name } \\
(1)\end{array}$ & $\begin{array}{l}\mathrm{z}_{1} \\
(2)\end{array}$ & $\begin{array}{c}\text { Reference } \\
(3)\end{array}$ & $\begin{array}{l}\mathrm{z}_{2} \\
(4)\end{array}$ \\
\hline \multicolumn{4}{|c|}{$4 \mathrm{FGL}$} \\
\hline 4FGL J0135.1+0255 & $\ldots$ & $\ldots$ & 0.372 \\
\hline 4FGL J0156.5+3914 & $\ldots$ & $\ldots$ & 0.446 \\
\hline 4FGL J1418.4+3543 & $\ldots$ & $\ldots$ & 0.819 \\
\hline 4FGL J2207.6+0053 & $\ldots$ & $\ldots$ & 0.970 \\
\hline \multicolumn{4}{|c|}{ Optical Campaign } \\
\hline J163738.24+300506.4 & $\ldots$ & Peña-Herazo et al. (2020) & 0.819 \\
\hline \multicolumn{4}{|c|}{ Roma BzCAT } \\
\hline 5BZQ J0010+1724 & 1.601 & Wills \& Wills (1976) & 0.769 \\
\hline 5BZB J0124+3249 & $\ldots$ & $\ldots$ & 0.780 \\
\hline 5BZB J0127-0151 & $\ldots$ & $\ldots$ & 0.337 \\
\hline 5BZB J0910+3902 & $\ldots$ & $\ldots$ & 0.199 \\
\hline 5BZQ J1047+2635 & 0.99 & Healey et al. (2008) & 2.560 \\
\hline 5BZB J1117+5355 & $\ldots$ & $\ldots$ & $>0.721$ \\
\hline 5BZB J1552+0850 & $\ldots$ & $\ldots$ & $>1.016$ \\
\hline 5BZB J1643+3221 & $\ldots$ & & $>1.029$ \\
\hline 5BZQ J1728+3838 & 1.386 & Monroe et al. (2016) & 0.630 \\
\hline $5 \mathrm{BZG}$ J2346+4024 & 0.0838 & Sowards-Emmerd et al. (2005) & 0.459 \\
\hline
\end{tabular}

Note: Columns (1) Source name ; (2) redshift in the sample catalog; (3) redshift reference; and,(4)redshift identified thanks to our analysis.

Blazars are the rarest class of active galactic nuclei and among them the subclass of BL Lacs is certainly the most elusive one (Urry \& Padovani 1995) even in the $\gamma$-ray sky where they constitute the largest known population of associated sources (Abdollahi et al. 2020).

Here we present the results of the analysis of 392 blazar spectra available in the archive of LAMOST, using its data release 5 . Sources were selected out of three main samples or catalogs, namely (1) the Fourth FermiLAT Point Source Catalog (Abdollahi et al. 2020), (2) the list of targets recently pointed during the follow up spectroscopic campaign of unidentified/unassociated $\gamma$-ray sources (Massaro et al. 2013b; D'Abrusco et al. 2014; Landoni et al. 2015; Ricci et al. 2015; Álvarez Crespo et al. 2016a,b; Peña-Herazo et al. 2017; Marchesini et al. 2019b; Peña-Herazo et al. 2019; de Menezes et al. 2020; Peña-Herazo et al. 2020), including results of other groups (e.g., Shaw et al. 2013; Klindt et al. 2017; Paiano et al. 2017a,b, 2019) and (3) the multifrequency catalog of blazars: the Roma-BZCAT (Massaro et al. 2009, 2015a). The total number of those lying in the LAMOST footprint and having an optical spectrum with a signal to noise ratio greater than 10 , thus allowing us to perform our investigation, are a total of 392. For $\sim 48 \%$, i.e., 188 out of 392 , we also compare the spectra available thanks to LAMOST with those collected in the SDSS.
From the 4FGL sample, we analyzed the spectra of 31 sources, including 20 BCUs, ten known blazars, and one AGN. Our largest sample, analyzed here, is constituted by 353 blazars in Roma-BZCAT, classified as 184 BZQs, 39 BZGs, and 130 BZBs. Our results are then summarized in Table 2, comparing the original classification with the classification presented in the following analysis.

- We classified 20 BCUs listed in the 4FGL and one AGN, all of unknown nature, confirming that they are all blazars. Given the large number (15 out of 19) BCUs that are indeed BZBs, we conclude that BL Lacs are the most elusive class of extragalactic $\gamma$-ray sources.

- We obtained a total of 15 new redshifts for blazars and previously uncertain blazars listed in our samples.

- We discovered 26 potential transitional (i.e., changing-look) blazars. In particular we found both BZBs and BZGs that were reclassified as BZQs as well as BZQs that showed a classical BZB featureless optical spectrum. Although, we did not find literature spectra to firmly classify them as changing-look. 
- We were also able to confirm the blazar-like nature of six unconfirmed BL Lac listed in the 4FGL.

All the remaining sources analyzed are in agreement with previous literature classifications. In particular the redshifts for six BZBs, all from the Roma-BZCAT sample, are listed here: 5BZB J0124+3249 at $z=0.780$, 5BZB J0127-0151 at $z=0.337,5$ BZB J0910+3902 at $z=0.199,5 \mathrm{BZB} \mathrm{J} 1117+5355$ at $z=0.721,5 \mathrm{BZB}$ $\mathrm{J} 1552+0850$ at $z=1.016$, and, 5BZB J1643+3221 at $z=1.029$, since they all lie in the tail of high redshift BL Lac population (Landoni et al. 2018) but consistent with the whole distribution as in Roma-BZCAT and similar to those arising from our optical spectroscopic campaign (Peña-Herazo et al. 2020).

From the comparison of the literature spectral classification with our classification based on LAMOST spectra we found a difference in classification for 26 objects. We summarize these results in Table 3, showing their original classification and its reference. Among these 26 blazars we confirm the changing-look nature of J140244.51+155956.6. We present the comparison of the literature spectrum with the LAMOST and SDSS spectra. In Figure 2, we also present other potential changing-look blazars for which we found literature spectra. Studying the population of changing-look blazars is of crucial importance for avoiding selection effects due to miss classify objects for studying the cosmic evolution of blazars. This sample of changing-look blazars can be of interest of spectral variability studies as the Time Domain Spectroscopic Survey (Green et al. 2014; MacLeod et al. 2018).

LAMOST survey revealed to be an extremely useful tool, previously unexplored, to investigate blazars, their variability, classification and redshift measurement.

\section{ACKNOWLEDGMENTS}

H.P.-H. and V.C. acknowledge support from CONACyT research grant No. 280789. MFG acknowledges support from the National Science Foundation of China (grant 11873073). This work is supported by the "Departments of Excellence 2018 - 2022" Grant awarded by the Italian Ministry of Education, University and Research (MIUR) (L. 232/2016). This research has made use of resources provided by the Ministry of Education, Universities and Research for the grant MASF_FFABR_17_01. A.P. acknowledges financial support from the Consorzio Interuniversitario per la fisica Spaziale (CIFS) under the agreement related to the grant MASF_CONTR_FIN_18_02.
This work is based on data from Guoshoujing Telescope (the Large Sky Area Multi-Object Fiber Spectroscopic Telescope LAMOST), is a National Major Scientific Project built by the Chinese Academy of Sciences. Funding for the project has been provided by the National Development and Reform Commission. LAMOST is operated and managed by the National Astronomical Observatories, Chinese Academy of Sciences. Part of this work is based on archival data, software or on-line services provided by the ASI Science Data Center. the SIMBAD database operated at CDS, Strasbourg, France; the NASA/IPAC Extragalactic Database (NED) operated by the Jet Propulsion Laboratory, California Institute of Technology, under contract with the National Aeronautics and Space Administration. This publication makes use of data products from the Wide-field Infrared Survey Explorer, which is a joint project of the University of California, Los Angeles, and the Jet Propulsion Laboratory/California Institute of Technology, funded by the National Aeronautics and Space Administration. Funding for the SDSS and SDSS-II has been provided by the Alfred P. Sloan Foundation, the Participating Institutions, the National Science Foundation, the U.S. Department of Energy, the National Aeronautics and Space Administration, the Japanese Monbukagakusho, the Max Planck Society, and the Higher Education Funding Council for England. The SDSS Web Site is http://www.sdss.org/. The SDSS is managed by the Astrophysical Research Consortium for the Participating Institutions. The Participating Institutions are the American Museum of Natural History, Astrophysical Institute Potsdam, University of Basel, University of Cambridge, Case Western Reserve University, University of Chicago, Drexel University, Fermilab, the Institute for Advanced Study, the Japan Participation Group, Johns Hopkins University, the Joint Institute for Nuclear Astrophysics, the Kavli Institute for Particle Astrophysics and Cosmology, the Korean Scientist Group, the Chinese Academy of Sciences (LAMOST), Los Alamos National Laboratory, the Max-Planck-Institute for Astronomy (MPIA), the MaxPlanck-Institute for Astrophysics (MPA), New Mexico State University, Ohio State University, University of Pittsburgh, University of Portsmouth, Princeton University, the United States Naval Observatory, and the University of Washington. TOPCAT ${ }^{1}$ (Taylor 2005) for the preparation and manipulation of the tabular data and the images.

\footnotetext{
${ }^{1}$ http://www.star.bris.ac.uk/ mbt/topcat/
} 


\section{REFERENCES}

Abdo, A. A., Ackermann, M., Agudo, I., et al. 2010a, ApJ, 716, 30, doi: 10.1088/0004-637X/716/1/30

Abdo, A. A., Ackermann, M., Ajello, M., et al. 2010b, ApJ, 715, 429, doi: 10.1088/0004-637X/715/1/429

—. 2010c, ApJS, 188, 405, doi: 10.1088/0067-0049/188/2/405

Abdollahi, S., Acero, F., Ackermann, M., et al. 2020, ApJS, 247, 33, doi: 10.3847/1538-4365/ab6bcb

Acciari, V. A., Aliu, E., Beilicke, M., et al. 2010, ApJL, 709, L163, doi: 10.1088/2041-8205/709/2/L163

Acero, F., Donato, D., Ojha, R., et al. 2013, ApJ, 779, 133, doi: 10.1088/0004-637X/779/2/133

Acero, F., Ackermann, M., Ajello, M., et al. 2015, ApJS, 218, 23, doi: 10.1088/0067-0049/218/2/23

Ackermann, M., Ajello, M., Allafort, A., et al. 2012a, ApJ, 753, 83, doi: 10.1088/0004-637X/753/1/83

—. 2012b, Science, 338, 1190, doi: 10.1126/science. 1227160

Ackermann, M., Albert, A., Anderson, B., et al. 2014, PhRvD, 89, 042001, doi: 10.1103/PhysRevD.89.042001

Ackermann, M., Ajello, M., Atwood, W. B., et al. 2015, ApJ, 810, 14, doi: 10.1088/0004-637X/810/1/14

—. 2016, ApJS, 222, 5, doi: 10.3847/0067-0049/222/1/5

Acosta-Pulido, J., Castro Segura, N., Carnerero, M., \& Raiteri, C. 2017, Galaxies, 5, 1, doi: 10.3390/galaxies5010001

Aguado, D. S., Ahumada, R., Almeida, A., et al. 2019, ApJS, 240, 23, doi: 10.3847/1538-4365/aaf651

Aharonian, F., Akhperjanian, A. G., Bazer-Bachi, A. R., et al. 2007, ApJL, 664, L71, doi: 10.1086/520635

Ajello, M., Romani, R. W., Gasparrini, D., et al. 2014, ApJ, 780, 73, doi: 10.1088/0004-637X/780/1/73

Albert, J., Aliu, E., Anderhub, H., et al. 2007, ApJ, 669, 862, doi: $10.1086 / 521382$

Álvarez Crespo, N., Masetti, N., Ricci, F., et al. 2016a, AJ, 151, 32, doi: 10.3847/0004-6256/151/2/32

Álvarez Crespo, N., Massaro, F., Milisavljevic, D., et al. 2016b, AJ, 151, 95, doi: 10.3847/0004-6256/151/4/95

Álvarez Crespo, N., Massaro, F., D’Abrusco, R., et al. 2016c, Ap\&SS, 361, 316, doi: 10.1007/s10509-016-2902-1

Amaya-Almazán, R. A., Chavushyan, V., \& Patiño-Álvarez, V. M. 2021, ApJ, 906, 5, doi: 10.3847/1538-4357/abc689

Archambault, S., Archer, A., Beilicke, M., et al. 2015, ApJ, 808, 110, doi: 10.1088/0004-637X/808/2/110

Arsioli, B., Fraga, B., Giommi, P., Padovani, P., \& Marrese, P. M. 2015, A\&A, 579, A34, doi: 10.1051/0004-6361/201424148

Atwood, W. B., Abdo, A. A., Ackermann, M., et al. 2009, ApJ, 697, 1071, doi: 10.1088/0004-637X/697/2/1071
Baldwin, J. A., Wampler, E. J., Burbidge, E. M., et al. 1977, ApJ, 215, 408, doi: 10.1086/155370

Berlin, A., \& Hooper, D. 2014, PhRvD, 89, 016014, doi: 10.1103/PhysRevD.89.016014

Bianchin, V., Foschini, L., Ghisellini, G., et al. 2009, A\&A, 496, 423, doi: 10.1051/0004-6361/200811128

Blandford, R. D., \& Rees, M. J. 1978, in BL Lac Objects, ed. A. M. Wolfe, 328-341

Bruni, G., Panessa, F., Ghisellini, G., et al. 2018, ApJL, 854, L23, doi: 10.3847/2041-8213/aaacfb

Capetti, A., Raiteri, C. M., \& Buttiglione, S. 2010, A\&A, 516, A59, doi: 10.1051/0004-6361/201014232

Chatterjee, R., Bailyn, C. D., Bonning, E. W., et al. 2012, ApJ, 749, 191, doi: 10.1088/0004-637X/749/2/191

Chavushyan, V., Patiño-Álvarez, V. M., Amaya-Almazán, R. A., \& Carrasco, L. 2020, ApJ, 891, 68, doi: 10.3847/1538-4357/ab6ef6

Cheung, C. C., Donato, D., Gehrels, N., Sokolovsky, K. V., \& Giroletti, M. 2012, ApJ, 756, 33, doi: 10.1088/0004-637X/756/1/33

Cowperthwaite, P. S., Massaro, F., D'Abrusco, R., et al. 2013, AJ, 146, 110, doi: 10.1088/0004-6256/146/5/110

Cui, X.-Q., Zhao, Y.-H., Chu, Y.-Q., et al. 2012, Research in Astronomy and Astrophysics, 12, 1197, doi: 10.1088/1674-4527/12/9/003

D'Abrusco, R., Massaro, F., Ajello, M., et al. 2012, ApJ, 748, 68, doi: 10.1088/0004-637X/748/1/68

D'Abrusco, R., Massaro, F., Paggi, A., et al. 2013, ApJS, 206, 12, doi: 10.1088/0067-0049/206/2/12

—. 2014, ApJS, 215, 14, doi: 10.1088/0067-0049/215/1/14

D'Abrusco, R., Álvarez Crespo, N., Massaro, F., et al. 2019, ApJS, 242, 4, doi: 10.3847/1538-4365/ab16f4

de Menezes, R., Peña-Herazo, H. A., Marchesini, E. J., et al. 2019, A\&A, 630, A55, doi: 10.1051/0004-6361/201936195

de Menezes, R., Amaya-Almazán, R. A., Marchesini, E. J., et al. 2020, Ap\&SS, 365, 12, doi: 10.1007/s10509-020-3727-5

Desai, A., Marchesi, S., Rajagopal, M., \& Ajello, M. 2019, ApJS, 241, 5, doi: 10.3847/1538-4365/ab01fc

Doert, M., \& Errando, M. 2014, ApJ, 782, 41, doi: 10.1088/0004-637X/782/1/41

Domínguez Sánchez, H., Pozzi, F., Gruppioni, C., et al. 2011, MNRAS, 417, 900, doi: 10.1111/j.1365-2966.2011.19263.x

Evans, I. N., \& Koratkar, A. P. 2004, ApJS, 150, 73, doi: $10.1086 / 379649$

Fan, J. H., Yang, J. H., Liu, Y., et al. 2016, ApJS, 226, 20, doi: $10.3847 / 0067-0049 / 226 / 2 / 20$ 
Fischer, J. U., Hasinger, G., Schwope, A. D., et al. 1998, Astronomische Nachrichten, 319, 347, doi: 10.1002/asna.2123190603

Fossati, G., Maraschi, L., Celotti, A., Comastri, A., \& Ghisellini, G. 1998, MNRAS, 299, 433, doi: 10.1046/j.1365-8711.1998.01828.x

Gaur, H., Gupta, A. C., Strigachev, A., et al. 2012, MNRAS, 425, 3002, doi: 10.1111/j.1365-2966.2012.21583.x

Ghisellini, G., Tavecchio, F., Foschini, L., \& Ghirlanda, G. 2011, MNRAS, 414, 2674, doi: 10.1111/j.1365-2966.2011.18578.x

Ghisellini, G., Tavecchio, F., Foschini, L., et al. 2012, MNRAS, 425, 1371, doi: 10.1111/j.1365-2966.2012.21554.x

Giannios, D., Uzdensky, D. A., \& Begelman, M. C. 2009, MNRAS, 395, L29, doi: 10.1111/j.1745-3933.2009.00635.x

Giommi, P., Ansari, S. G., \& Micol, A. 1995, A\&AS, 109, 267

Giommi, P., \& Padovani, P. 2015, MNRAS, 450, 2404, doi: $10.1093 /$ mnras/stv793

Giommi, P., Padovani, P., \& Polenta, G. 2013, MNRAS, 431, 1914, doi: 10.1093/mnras/stt305

Giommi, P., Padovani, P., Polenta, G., et al. 2012, MNRAS, 420, 2899, doi: 10.1111/j.1365-2966.2011.20044.x

Giroletti, M., Massaro, F., D'Abrusco, R., et al. 2016, Astronomy \& Astrophysics, 588, A141

Green, P. J., Anderson, S. F., Morganson, E., et al. 2014, in American Astronomical Society Meeting Abstracts, Vol. 223, American Astronomical Society Meeting Abstracts \#223, 116.15

Gu, M. F., \& Ai, Y. L. 2011, A\&A, 528, A95, doi: 10.1051/0004-6361/201016280

Gupta, A. 2018, Galaxies, 6, 1, doi: 10.3390/galaxies6010001

Hayashida, M., Nalewajko, K., Madejski, G. M., et al. 2015, ApJ, 807, 79, doi: 10.1088/0004-637X/807/1/79

Healey, S. E., Romani, R. W., Taylor, G. B., et al. 2007, ApJS, 171, 61, doi: 10.1086/513742

Healey, S. E., Romani, R. W., Cotter, G., et al. 2008, ApJS, 175, 97, doi: 10.1086/523302

Hewitt, A., \& Burbidge, G. 1991, ApJS, 75, 297, doi: 10.1086/191533

Isler, J. C., Urry, C. M., Coppi, P., et al. 2013, ApJ, 779, 100, doi: 10.1088/0004-637X/779/2/100

Jones, D. H., Saunders, W., Colless, M., et al. 2004, MNRAS, 355, 747, doi: 10.1111/j.1365-2966.2004.08353.x

Jones, D. H., Read, M. A., Saunders, W., et al. 2009, MNRAS, 399, 683, doi: 10.1111/j.1365-2966.2009.15338.x
Jorstad, S. G., Marscher, A. P., Mattox, J. R., et al. 2001, ApJS, 134, 181, doi: 10.1086/320858

Jorstad, S. G., Marscher, A. P., Smith, P. S., et al. 2013, ApJ, 773, 147, doi: 10.1088/0004-637X/773/2/147

Kaur, A., Falcone, A. D., Stroh, M. D., Kennea, J. A., \& Ferrara, E. C. 2019, ApJ, 887, 18, doi: $10.3847 / 1538-4357 /$ ab4ceb

Kaur, A., Rau, A., Ajello, M., et al. 2018, ApJ, 859, 80, doi: 10.3847/1538-4357/aabdec

Kellermann, K. I., Kovalev, Y. Y., Lister, M. L., et al. 2007, Ap\&SS, 311, 231, doi: 10.1007/s10509-007-9622-5

Kerr, M. 2019, ApJ, 885, 92, doi: 10.3847/1538-4357/ab459f

Klindt, L., van Soelen, B., Meintjes, P. J., \& Väisänen, P. 2017, MNRAS, 467, 2537, doi: 10.1093/mnras/stx218

Kovalev, Y. Y. 2009, ApJL, 707, L56, doi: 10.1088/0004-637X/707/1/L56

Landi, R., Bassani, L., Stephen, J. B., et al. 2015, A\&A, 581, A57, doi: 10.1051/0004-6361/201526221

Landoni, M., Falomo, R., Paiano, S., \& Treves, A. 2020, ApJS, 250, 37, doi: 10.3847/1538-4365/abb5ae

Landoni, M., Paiano, S., Falomo, R., Scarpa, R., \& Treves, A. 2018, ApJ, 861, 130, doi: 10.3847/1538-4357/aac77c

Landoni, M., Massaro, F., Paggi, A., et al. 2015, AJ, 149, 163, doi: 10.1088/0004-6256/149/5/163

Landt, H., Padovani, P., \& Giommi, P. 2002, MNRAS, 336, 945, doi: 10.1046/j.1365-8711.2002.05832.x

León-Tavares, J., Chavushyan, V., Patiño-Álvarez, V., et al. 2013, ApJL, 763, L36, doi: 10.1088/2041-8205/763/2/L36

Liodakis, I., \& Blinov, D. 2019, MNRAS, 486, 3415, doi: $10.1093 / \mathrm{mnras} / \mathrm{stz} 1008$

Lister, M. L., Aller, M. F., Aller, H. D., et al. 2013, AJ, 146, 120, doi: 10.1088/0004-6256/146/5/120

Liu, X., Yang, P. P., Liu, J., et al. 2017, MNRAS, 469, 2457, doi: 10.1093/mnras/stx1062

MacLeod, C. L., Green, P. J., Anderson, S. F., et al. 2018, AJ, 155, 6, doi: 10.3847/1538-3881/aa99da

Mandarakas, N., Blinov, D., Liodakis, I., et al. 2019, A\&A, 623, A61, doi: 10.1051/0004-6361/201834458

Mao, P., Urry, C. M., Massaro, F., et al. 2016, ApJS, 224, 26, doi: 10.3847/0067-0049/224/2/26

Marchesini, E. J., Paggi, A., Massaro, F., et al. 2020, A\&A, 638, A128, doi: 10.1051/0004-6361/201936928

-. 2019a, A\&A, 631, A150, doi: 10.1051/0004-6361/201936285

Marchesini, E. J., Peña-Herazo, H. A., Álvarez Crespo, N., et al. 2019b, Ap\&SS, 364, 5, doi: $10.1007 / \mathrm{s} 10509-018-3490-\mathrm{z}$

Massaro, E., Giommi, P., Leto, C., et al. 2009, A\&A, 495, 691, doi: 10.1051/0004-6361:200810161 
Massaro, E., Maselli, A., Leto, C., et al. 2015a, Ap\&SS, 357, 75, doi: 10.1007/s10509-015-2254-2

Massaro, E., Nesci, R., \& Piranomonte, S. 2012a, MNRAS, 422, 2322, doi: 10.1111/j.1365-2966.2012.20782.x

Massaro, F., \& D'Abrusco, R. 2016, ApJ, 827, 67, doi: 10.3847/0004-637X/827/1/67

Massaro, F., D’Abrusco, R., Ajello, M., Grindlay, J. E., \& Smith, H. A. 2011, ApJL, 740, L48, doi: 10.1088/2041-8205/740/2/L48

Massaro, F., D'Abrusco, R., Giroletti, M., et al. 2013, The Astrophysical Journal Supplement Series, 207, 4

Massaro, F., D'Abrusco, R., Paggi, A., et al. 2013a, ApJS, 206, 13, doi: 10.1088/0067-0049/206/2/13

—. 2013b, ApJS, 209, 10, doi: 10.1088/0067-0049/209/1/10

Massaro, F., D'Abrusco, R., Tosti, G., et al. 2012b, ApJ, 750, 138, doi: 10.1088/0004-637X/750/2/138

—. 2012c, ApJ, 752, 61, doi: 10.1088/0004-637X/752/1/61

Massaro, F., Landoni, M., D'Abrusco, R., et al. 2015b, A\&A, 575, A124, doi: 10.1051/0004-6361/201425119

Massaro, F., Marchesini, E. J., D'Abrusco, R., et al. 2017, ApJ, 834, 113, doi: 10.3847/1538-4357/834/2/113

Massaro, F., Masetti, N., D’Abrusco, R., Paggi, A., \& Funk, S. 2014, AJ, 148, 66, doi: 10.1088/0004-6256/148/4/66

Massaro, F., Paggi, A., Errando, M., et al. 2013c, ApJS, 207, 16, doi: 10.1088/0067-0049/207/1/16

Massaro, F., Thompson, D. J., \& Ferrara, E. C. 2015c, A\&A Rv, 24, 2, doi: 10.1007/s00159-015-0090-6

Massaro, F., D'Abrusco, R., Landoni, M., et al. 2015d, ApJS, 217, 2, doi: 10.1088/0067-0049/217/1/2

Massaro, F., Álvarez Crespo, N., D’Abrusco, R., et al. 2016a, Ap\&SS, 361, 337, doi: 10.1007/s10509-016-2926-6

—. 2016b, Ap\&SS, 361, 337, doi: 10.1007/s10509-016-2926-6

Monroe, T. R., Prochaska, J. X., Tejos, N., et al. 2016, AJ, 152, 25, doi: 10.3847/0004-6256/152/1/25

Nalewajko, K., Gupta, A. C., Liao, M., et al. 2019, A\&A, 631, A4, doi: 10.1051/0004-6361/201935904

Nass, P., Bade, N., Kollgaard, R. I., et al. 1996, A\&A, 309, 419

Nolan, P. L., Abdo, A. A., Ackermann, M., et al. 2012, ApJS, 199, 31, doi: 10.1088/0067-0049/199/2/31

Nori, M., Giroletti, M., Massaro, F., et al. 2014, The Astrophysical Journal Supplement Series, 212, 3

Paggi, A., Massaro, F., D'Abrusco, R., et al. 2013, ApJS, 209, 9, doi: 10.1088/0067-0049/209/1/9

Paggi, A., Milisavljevic, D., Masetti, N., et al. 2014, AJ, 147, 112, doi: 10.1088/0004-6256/147/5/112

Paiano, S., Falomo, R., Franceschini, A., Treves, A., \& Scarpa, R. 2017a, ApJ, 851, 135, doi: 10.3847/1538-4357/aa9af4
Paiano, S., Falomo, R., Treves, A., Franceschini, A., \& Scarpa, R. 2019, ApJ, 871, 162, doi: 10.3847/1538-4357/aaf6e4

Paiano, S., Falomo, R., Treves, A., \& Scarpa, R. 2020, MNRAS, 497, 94, doi: 10.1093/mnras/staa1840

Paiano, S., Landoni, M., Falomo, R., Treves, A., \& Scarpa, R. 2017b, ApJ, 844, 120, doi: 10.3847/1538-4357/aa7aac

Paliya, V. S., Stalin, C. S., Ajello, M., \& Kaur, A. 2017, ApJ, 844, 32, doi: 10.3847/1538-4357/aa77f5

Park, J., Kam, M., Trippe, S., et al. 2018, ApJ, 860, 112, doi: 10.3847/1538-4357/aac490

Patiño-Álvarez, V. M., Fernandes, S., Chavushyan, V., et al. 2017, Frontiers in Astronomy and Space Sciences, 4, 47, doi: 10.3389/fspas.2017.00047

Peña-Herazo, H. A., Marchesini, E. J., Álvarez Crespo, N., et al. 2017, Ap\&SS, 362, 228, doi: 10.1007/s10509-017-3208-7

Peña-Herazo, H. A., Massaro, F., Chavushyan, V., et al. 2019, Ap\&SS, 364, 85, doi: 10.1007/s10509-019-3574-4

Peña-Herazo, H. A., Amaya-Almazán, R. A., Massaro, F., et al. 2020, A\&A, 643, A103, doi: 10.1051/0004-6361/202037978

Petrov, L., Mahony, E. K., Edwards, P. G., et al. 2013, MNRAS, 432, 1294, doi: 10.1093/mnras/stt550

Pian, E., Urry, C. M., Maraschi, L., et al. 1999, ApJ, 521, 112, doi: 10.1086/307548

Poutanen, J. 1994, ApJS, 92, 607, doi: 10.1086/192024

Ricci, F., Massaro, F., Landoni, M., et al. 2015, AJ, 149, 160, doi: 10.1088/0004-6256/149/5/160

Ruan, J. J., Anderson, S. F., Plotkin, R. M., et al. 2014, ApJ, 797, 19, doi: 10.1088/0004-637X/797/1/19

Sabbey, C. N., Oemler, A., Coppi, P., et al. 2001, ApJ, 548, 585, doi: 10.1086/319036

Salvetti, D., Chiaro, G., La Mura, G., \& Thompson, D. J. 2017, MNRAS, 470, 1291, doi: 10.1093/mnras/stx1328

Sandrinelli, A., Treves, A., Falomo, R., et al. 2013, AJ, 146, 163, doi: 10.1088/0004-6256/146/6/163

Sarkar, A., Chitnis, V. R., Gupta, A. C., et al. 2019, ApJ, 887, 185, doi: 10.3847/1538-4357/ab5281

Schinzel, F. K., Petrov, L., Taylor, G. B., \& Edwards, P. G. 2017, ApJ, 838, 139, doi: 10.3847/1538-4357/aa6439

Schinzel, F. K., Petrov, L., Taylor, G. B., et al. 2015, ApJS, 217, 4, doi: 10.1088/0067-0049/217/1/4

Shaw, M. S., Romani, R. W., Cotter, G., et al. 2012, ApJ, 748, 49, doi: 10.1088/0004-637X/748/1/49

—. 2013, ApJ, 764, 135, doi: 10.1088/0004-637X/764/2/135

Sowards-Emmerd, D., Romani, R. W., Michelson, P. F., Healey, S. E., \& Nolan, P. L. 2005, ApJ, 626, 95, doi: $10.1086 / 429902$ 
Stickel, M., Padovani, P., Urry, C. M., Fried, J. W., \& Kuehr, H. 1991, ApJ, 374, 431, doi: 10.1086/170133

Stocke, J. T., Morris, S. L., Gioia, I. M., et al. 1991, ApJS, 76, 813, doi: 10.1086/191582

Stocke, J. T., \& Rector, T. A. 1997, ApJL, 489, L17, doi: 10.1086/310962

Su, D. Q., Cui, X., Wang, Y., \& Yao, Z. 1998, in Society of Photo-Optical Instrumentation Engineers (SPIE)

Conference Series, Vol. 3352, Advanced Technology Optical/IR Telescopes VI, ed. L. M. Stepp, 76-90, doi: $10.1117 / 12.319250$

Takeuchi, Y., Kataoka, J., Maeda, K., et al. 2013, ApJS, 208, 25, doi: 10.1088/0067-0049/208/2/25

Taylor, M. B. 2005, in Astronomical Society of the Pacific Conference Series, Vol. 347, Astronomical Data Analysis Software and Systems XIV, ed. P. Shopbell, M. Britton, \& R. Ebert, 29

Tody, D. 1986, in Society of Photo-Optical Instrumentation Engineers (SPIE) Conference Series, Vol. 627, Instrumentation in astronomy VI, ed. D. L. Crawford, 733, doi: $10.1117 / 12.968154$
Urry, C. M., \& Padovani, P. 1995, PASP, 107, 803, doi: $10.1086 / 133630$

Vermeulen, R. C., Ogle, P. M., Tran, H. D., et al. 1995, ApJL, 452, L5, doi: 10.1086/309716

Wills, D., \& Wills, B. J. 1976, ApJS, 31, 143, doi: 10.1086/190378

Wu, J., Vanden Berk, D., Grupe, D., et al. 2012, ApJS, 201, 10, doi: 10.1088/0067-0049/201/2/10

Yao, S., Wu, X.-B., Ai, Y. L., et al. 2019, ApJS, 240, 6, doi: 10.3847/1538-4365/aaef88

Zechlin, H. S., Fernandes, M. V., Elsässer, D., \& Horns, D. 2012, A\&A, 538, A93, doi: 10.1051/0004-6361/201117655

Zhang, H.-M., Wang, Z.-J., Zhang, J., et al. 2020, PASJ, 72, 44, doi: 10.1093/pasj/psaa029

Zhang, J., Liang, E.-W., Zhang, S.-N., \& Bai, J. M. 2012, ApJ, 752, 157, doi: 10.1088/0004-637X/752/2/157

Zhao, G., Zhao, Y.-H., Chu, Y.-Q., Jing, Y.-P., \& Deng, L.-C. 2012, Research in Astronomy and Astrophysics, 12, 723, doi: 10.1088/1674-4527/12/7/002 